\title{
Faraday Cup for Electron Flux Measurements on the Microtron MT 25
}

\author{
M. Vognar, С̌. Šimáně, D. Chvátil
}

The basic criteria for constructing of an evacuated Faraday cup for precise measurement of 5 to $25 \mathrm{MeV}$ electron beam currents in air from a microtron are established. The Faraday cup, built in the microtron laboratory of the Faculty of Nuclear Sciences and Physical Engineering of CTU Prague, is described together with the electronic chain and its incorporation in the measuring line on the beam. Measures to reduce the backward escape of electrons are explained. The range of currents is from $10^{-5}$ to $10^{-10} \mathrm{~A}$. The diameter of the Al entry window of the Faraday cup is $1.8 \mathrm{~cm}$, and its area is $2.54 \mathrm{~cm}^{2}$. The thickness of the entry window is $0.1 \mathrm{~mm}$.

Keywords: microtron, Faraday cup, electron beams, electron flux measurement

\section{Introduction}

The study of electron induced radiation effects requires a good knowledge of the value of electron flux density during irradiation. Interest in irradiation by electrons ranges from medical applications in oncology to investigations of radiation induced changes or radiation hardness of electronic solid state devices and other materials. The total beam intensity, or the number of electrons exiting from an accelerator, are normally monitored by methods - preferably not perturbing the beam - such as induction pick-up current meters situated at the vacuum side close to the beam exit window. All such devices require calibration by an absolute metering system that registers the total electron flux. If a simple metallic block is used to stop all electrons, the measured value of the current flowing from the block to the ground is normally lower than the real value, because some of the electrons are scattered backwards.

A well known device for eliminating of this effect is the Faraday cup. A typical Faraday cup is a cylinder made of conducting material with an inner cavity provided with a small input opening, giving little chance for electrons scattered back on the inner cavity walls to escape through it.

Some precautions need to be taken, if the electron energy is in the $\mathrm{MeV}$ range. The walls of the inner cavity must be thicker than the range of the most energetic electrons, so that none of the electrons entering the cavity can pass through. The walls should be made of low Z material, (e.g. graphite). This prefers the small angle scattering, and ensures that radiation processes accompanied by wide angle scattering of electrons and by emission of bremmsstrahlung, leading to photonuclear reactions, are reduced as far as possible. Low $\mathrm{Z}$ materials normally have low density and it would require rather thick cavity walls to dissipate the electron energy completely. Therefore low $\mathrm{Z}$ material is used to reduce the electron energy to a value at which the energy of the emitted bremmsstrahlung is below the threshold for photonuclear reactions. The remaining electron energy is then absorbed by heavier material. In this way the overall dimensions of the Faraday cup can be limited.

In order to reduce the escape of electrons from the Faraday cup even further, the electrons, scattered on the cavity walls toward its opening enter a magnetic field, which deviates them and forces them to end on the cavity wall. A second measure consists in reducing the escape by placing a decelerating electric field in front of the input opening between an auxiliary electrode on potential that is negative with respect to the cup.

If the Faraday cup were to be left in air at atmospheric pressure, the ionized air would constitute a leak resistor parallel to the input range resistor of the measuring device. To eliminate this effect, both the Faraday cup and the decelerating electrode have to be placed in a vacuum housing. The vacuum is maintained continually during measurement below 1 Pa by a rotary oil vacuum pump.

\section{Design and construction}

A schematic view of a Faraday cup with a vacuum shell is shown in Fig.1. The cavity 1 is shaped inside the graphite cylinder 2, inserted in a steel stopper 3. To stop $25 \mathrm{MeV}$

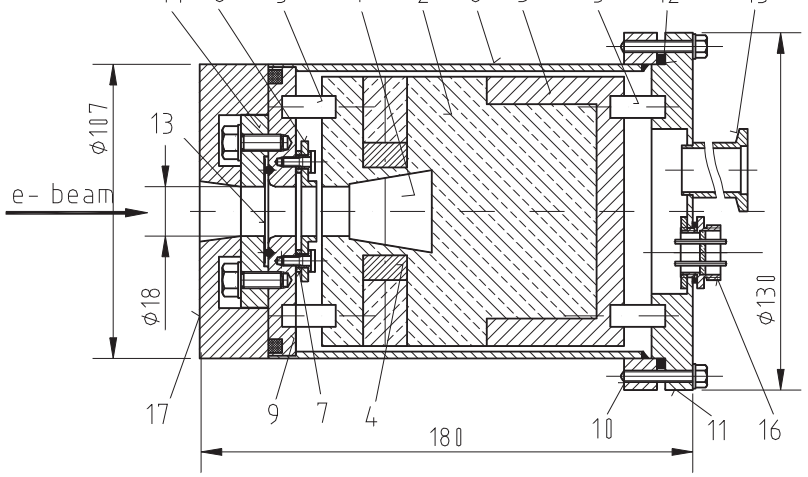

Fig. 1: Assembly drawing of the Faraday cup in the vacuum housing: 1 - cavity, 2 - graphite cup, 3 - steel stopper, 4 - permanent magnets, 5 - ceramic insulators, 6 - decelerating electrode, 7 - insulators, 8 - cylindrical part of the vacuum housing, 9 - housing bottom, 10 - collar, 11 - rear flange, 12 - rubber gasket, 13 - Al foil entry window, 14 - front flange, 15 - pumping sleeve, 16 - vacuum 4-lead bushing, 17 - Al front shielding

electrons completely would theoretically require about $14 \mathrm{~g} / \mathrm{cm}^{2}$ of graphite [1]. In our case the graphite thickness of $60 \mathrm{~mm}$ in the forward direction is followed by a $10 \mathrm{~mm}$ steel stopper, giving a total of $18 \mathrm{~g} / \mathrm{cm}^{2}$, guaranteeing fully zero escape of electrons through the walls of the cup. Two 


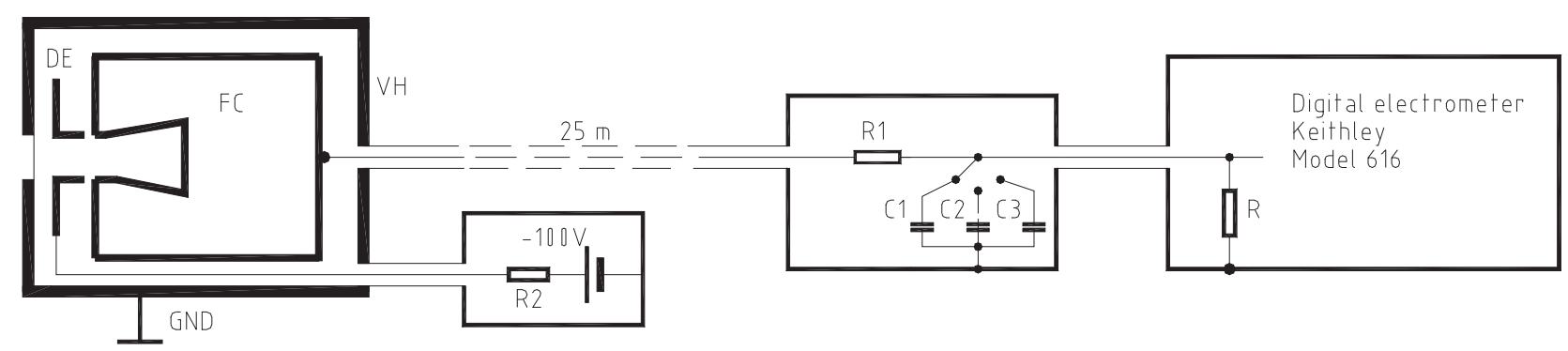

Fig. 2: Block scheme of the Faraday cup including the electrical measuring chain: FC - Faraday cup, DE - decelerating electrode, $\mathrm{VH}$ - vacuum housing, R1 - 0.2 M $\Omega, \mathrm{R} 2-0.5 \mathrm{M} \Omega, \mathrm{R}$ - electrometer input range resistor $10^{5}-10^{11} \Omega, \mathrm{C} 1-4.7 \mu \mathrm{F}, \mathrm{C} 2-0.1 \mu \mathrm{F}$, $\mathrm{C} 3-1 \mathrm{nF}$

permanent magnets 4 are inserted in part 2. The vacuum housing consists of a cylindrical part 6 with a collar 10 and a $10 \mathrm{~mm}$ thick steel bottom 9 . A rubber gasket 12 between the rear flange 11 and the collar 10 secures vacuum tightness of the housing. The Faraday cup is mounted on ceramic insulators 7 attached to the bottom 9 and the rear flange 11 . The $0.1 \mathrm{~mm}$ thick, $\Phi=18 \mathrm{~mm}$, Al entry window 13 is clamped between the $10 \mathrm{~mm}$ thick front flange 14 and the bottom 9 on a $\mathrm{Pb}$ ring seal. The decelerating electrode 6 is attached to the bottom on insulators 8 . The additional $25 \mathrm{~mm}$ thick $\mathrm{Al}$ front shielding 17 guarantees that no electrons enter the Faraday cup by any other way than through the $\mathrm{Al}$ entry window.

The electrical scheme of the Faraday cup and of the measuring chain, as well as the negative potential supply for the decelerating electrode, are shown in Fig. 2. The negative $-100 \mathrm{~V}$ potential for the decelerating electrode $D E$ is supplied by shielded coaxial cables from a dry battery installed in a shielded box. The resistor $R 1$ prevents a battery discharge in case of an accidental short circuit. A $25 \mathrm{~m}$ long coaxial cable is used to interconnect of the Faraday cup $F C$ and the input resistor $R$ of a 616 Keithley Digital Electrometer, operated as an amperemeter. The input of the electrometer is protected by resistor $R 1$. Because the electron beam can be considered as a current source with practically infinite inner resistance, the value of $R 1$ does not influence the measured current value. The input range resistor $R$ can be set in decade steps from $10^{5}$ to $10^{11} \Omega$ to give $1 \mathrm{~V}$ input voltage for currents from $10^{-5}$ to $10^{-11} \mathrm{~A}$, large enough to be well above the induced noise from high frequency electromagnetic and steady current sources in the microtron room. The shielding of the coaxial cable is connected to the housing of the Faraday cup. The resistor $R 1$ and $R$ between the Faraday cup and the housing, as well as the grounding of the housing to the laboratory earth (which is the only grounded point of the entire measuring chain) must never be disconnected. If this were to happen, the cup and the entire measuring chain would be brought to very high potential against the laboratory earth by the electric charge supplied by the high energy electrons, which would damage the electronic equipment.

The approximately rectangular electron beam pulses are $2.5 \mu$ s long with a repetition rate of $400 \mathrm{~Hz}$, the peak current value being 1000 times higher than the mean current value. To absorb the peaks and obtain the mean current value, the input time constant of the electrometer must be at least $0.1 \mathrm{~s}$. The capacity between the Faraday cup and the vacuum housing and the capacity of the coaxial cable are not sufficient to smooth the current, and extra capacitors must be connected parallel to the input resistor $R$, in order to obtain time constants between 0.1 and $10 \mathrm{~s}$. Capacitors $\mathrm{C} 1=4.7 \mu \mathrm{F}$, $\mathrm{C} 2=100 \mathrm{nF}$ and $\mathrm{C} 3=1 \mathrm{nF}$, in combination with input resistors from $10^{5}$ to $10^{10} \Omega$, are used to cover the current range from $10^{-5}$ to $10^{-10} \mathrm{~A}$. In any case, when the capacities need to be switched during the run of the microtron, they must not be disconnected in order to prevent the appearance of an excessive voltage on the input of the electrometer.

The accuracy of current measurements is limited by the value of the leak resistance between the Faraday cup and the earth, particularly the value of the resistance between the inner wire and the shielding of the $25 \mathrm{~m}$ long coaxial connecting cable. Its resistance of $1.5 \cdot 10^{11} \Omega$, parallel to the input range resistance $\mathrm{R}$, must be taken into account at currents lower than $10^{-10} \mathrm{~A}$.

The use of the Faraday cup in the study of problems connected with profiling electron fields for irradiation by scattering on foils are subject of a separate publication [3].

\section{Measuring arrangement}

An existing experimental installation (Fig. 3), which had been built to generate electron and photon fields for dosimetric purposes [2], was adapted to accommodate the Faraday cup. An optical bench 7, parallel to the beam and bearing the Faraday cup, was installed on a table top 8 . The table together with a system of diaphragms, rests on a common platform carriage 10 , which can be moved parallel to the beam on guide rails 11 . All the systems of the diaphragms and the Faraday cap are exactly centered by an optical laser and set in the beam axis by leveling with a survey theodolite. The distance between the beam exit window 1 and the entry window 2 of the Faraday cup can be changed from 860 to $1625 \mathrm{~mm}$ by a combination of shifting of the carriage on the guide rails and shifting the Faraday cup on the optical bench. By inserting diaphragms in the mounts of turrets 3 and 4 and in the conical steel collimator 5, one can set the beam aperture and modify by scattering the angular distribution of the electron flux density.

In the broad electron beam in air some of the scattered electrons can reach the Faraday cup through the side walls of the vacuum housing. The walls are not thick enough to prevent their penetration. To get the true value of the electron flux entering the cup by the entry window, this effect should be taken into account by multiplying the measured 


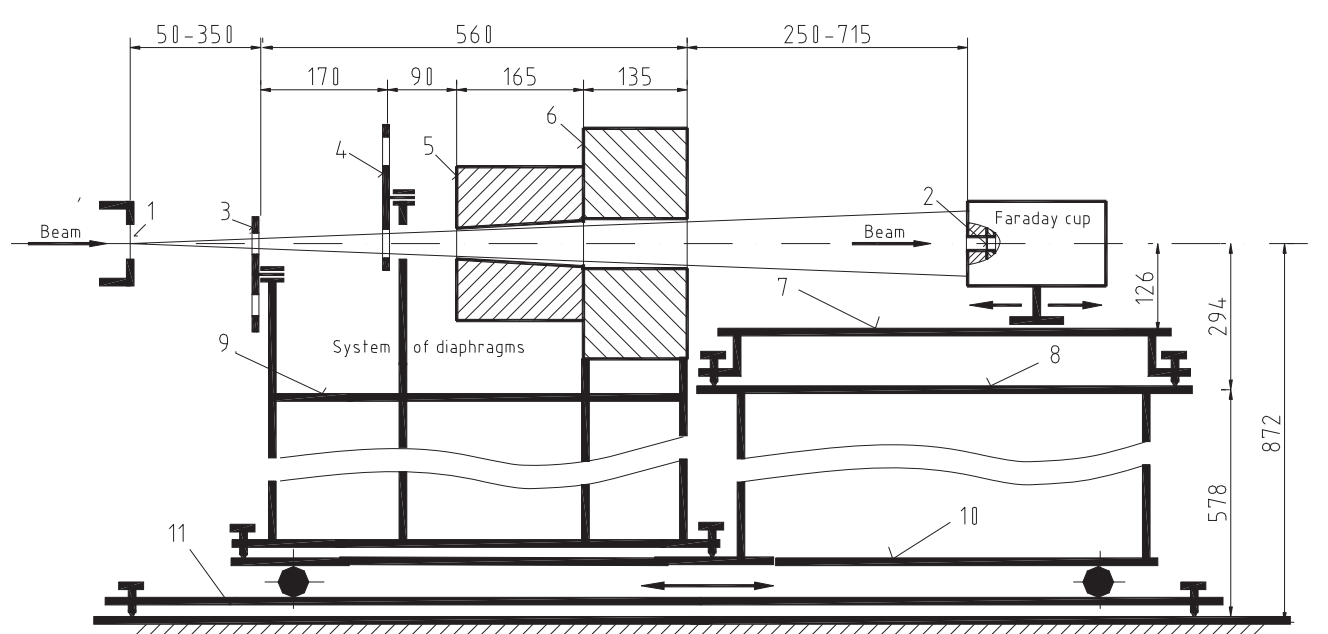

Fig. 3: Measuring line: 1 - electron beam exit window, 2 - Faraday cup entry window, 3 - first indexed turret with four diaphragm mounts, 4 - second indexed turret with eight diaphragm mounts, 5 - conical stainless steel collimator, 6 - square W-steel collimator, 7 - optical bench, 8 - table desk, 9 - diaphragm frame, 10 - common platform carriage, 11 - guide rail

electron flux by a coefficient, the value of which may be determined from the difference of the fluxes measured at the Faraday cup entry window when it is open and when it is blocked by a stopper. For example, at a distance $130 \mathrm{~cm}$ from the beam outlet window, at a beam aperture of $1.57^{\circ}$, the value of this coefficient is 0.93 , and at an aperture of $4.6^{\circ}$ the coefficient is equal to 0.88 .

\section{Conclusion}

The standard diameter of the electron beam exiting from the exit window of the microtron is of the order of 0.3 to $0.5 \mathrm{~cm}$. Its angular width is due mainly to the scattering on the $\mathrm{Al}$ exit window. Measured at the half heights of the peak value, it ranges from $2.2^{\circ}$ for $25 \mathrm{MeV}$ electrons to $5^{\circ}$ for electrons of $10 \mathrm{MeV}$ [3]. On the other hand, the angular aperture of the Faraday cup entry window at $5 \mathrm{~cm}$ distance from the exit window is about $20^{\circ}$, which is sufficient to receive the whole beam even in the worst case of $10 \mathrm{MeV}$ electrons. In this position the Faraday cup is used for absolute calibration of the beam exiting from the microtron in air. At a sufficiently large distance from the beam exit window (this distance depends on the angular width of the beam), the electron flux density passing through the entry window of the Faraday cup is practically uniform. At this distance one can determine the mean electron flux density (the number of electrons passing through $1 \mathrm{~cm}^{2}$ per second) at the maximum of the angular distribution by dividing the mean electron current value by the area of the window, which is $2.54 \mathrm{~cm}^{2}$, and by the electric charge of an electron $1.6 \cdot 10^{-19}$ C. The peak values of the electron flux densities in the pulse are a thousand times higher. At intermediate distances, one must take into consideration the angular distribution of the electron flux density in the beam. By dividing the measured mean electron current by the area of the entry window and by the electron charge, the mean electron flux density is obtained.

\section{References}

[1] Kovalev, V. P.: Vtoritchnye izlutchenija uskoritelej elektronov. Atomizdat, Moskva 1979

[2] Vognar, M., Šimáně, Č., Burian, A., Chvátil, D.: Electron and photon fields for dosimetric metrology generated by electron beams from a microtron. Nuclear Instruments and Methods in Physics Research A 380 (1996), pp. 613-617

[3] Šimáně, Č., Vognar, M., Chvátil, D.: Some Aspects of Profiling Electron Fields for Irradiation by Scattering on Foils. Submitted for publication in Acta Polytechnica

\footnotetext{
Ing. Miroslav Vognar

Prof. Ing. Čestmír Šimáně, DrSc.

Ing. David Chvátil

Dept. of Dosimetry \& Appl. of Ionizing Radiation

phone: +42022323657, +42022315212

fax: +42022320861

e-mail:Vognar@br.fjfi.cvut.cz

CTU, Faculty of Nuclear Science \& Physical Engineering

Břehová 7, 11519 Praha 1

Czech Republic
} 